\title{
Preliminary studies for three experiments at Treiman-Yang criterion
}

\author{
I.V. Kres ${ }^{1}$, V.N. Kondratyev ${ }^{1}$, S. Cherubini ${ }^{2}$ and C. Spitaleri ${ }^{2}$
}

1 Taras Shevchenko National University of Kyiv, Ukraine

${ }^{2}$ Dipartimento di Fisica e Astronomia, Università di Catania e INFN - Laboratori Nazionali del Sud, Catania, Italy

\begin{abstract}
Nuclear reactions with three bodies in their final state may proceed through different reaction mechanisms. The Feynman graph technique has been widely used to describe such reactions. However, it is very difficult in general to select the graphs that dominate in given process. The Treiman-Yang criterion is one of the most powerful experimental tests for verifying the pole approximation prediction when describing a quasi-free reaction mechanism. We propose the theoretical study of the $H^{2}\left(B^{10}, \alpha B e^{7}\right) n_{s}, H^{1}\left(B^{11}, \alpha_{1} \alpha_{2}\right) \alpha_{s}, H e^{3}\left(B e^{9}, \alpha_{1} \alpha_{2}\right) \alpha_{s}$ reactions at different energies. The preliminary study helps to check the existence of a QF channel by using the TY creterion.
\end{abstract}

The Trojah Horse Method (THM) is a powerful indirect technique that allows to determine the astrophysical factor for rearrangement reactions. The THM involves obtaining the cross section of the binary process $0+\mathrm{T} \rightarrow 1+2$ at astrophysical energies by measuring the Trojan Horse $(\mathrm{TH})$ reaction (the two-body to three-body process) $0+\mathrm{N} \rightarrow 1+2+\mathrm{s}$ in the quasifree $(\mathrm{QF})$ kinematics mode, where the Trojan Horse particle, $\mathrm{N}=(\mathrm{s}+\mathrm{T})$, which has a dominant cluster structure, is accelerated at energies above the Coulomb barrier.

The advantage of the THM is that the extracted cross section of the binary subprocess does not contain the Coulomb barrier suppresion factor and electron screening does not play a role in the cross section. After penetrating the barrier, the $\mathrm{TH}$-nucleus $\mathrm{N}$ undergoes breakup leaving particle $\mathrm{T}$ 


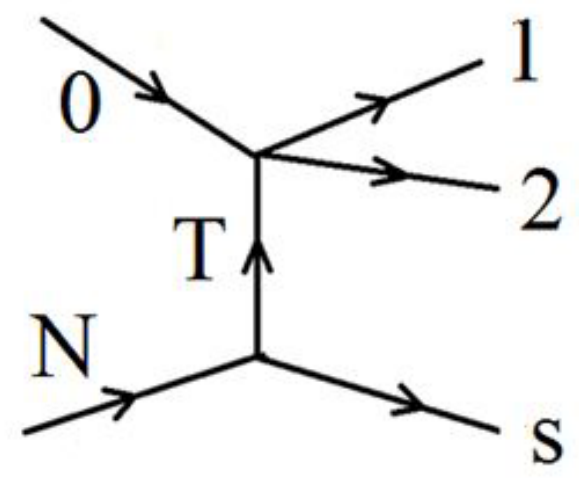

Figure 1: The diagram describing the $\mathrm{TH}$ reaction $0+\mathrm{N} \rightarrow 1+2+\mathrm{s}$ in the QF kinematics

to interact with particle 0 while particle s, also called spectator, flies away. From the measured cross section of TH reaction, the energy dependence of the binary sub-process is determined. The TH reaction mechanism shown schematically in fig.1, gives the dominant contribution to the cross section in a restricted region of the three-body phase space when the relative momentum of the fragments $\mathrm{s}$ and $\mathrm{T}$ is zero (the $\mathrm{QF}$ kinematical condition, when $l=0)$. [1]

Nuclear reactions with three bodies in their final state may proceed throught a variety of reaction mechanisms. In general the calculation of the reaction cross-section should include terms from quasi-free processes, expecially when appropriate regions of the phase-space are selected. Many models have been developed to describe such direct reactions. Among these the Feynman graph technique has been widely used but unfortunately it is very difficult in general to select the particular graphs that are dominant for a given process. In particular the role of the pole mechanism has been investigated in detail and attempts have been made to find sensitive criteria able to establish its relative importance. One of these criteria is the study of the distribution with respect to the Treiman-Yang angle. This criterion shows that under given conditions such a distribution should be isotropic, provided the pole machanism is dominant in reaction. The Treiman-Yang criterion is a necessary criterion but not a sufficient condition for dominance of the pole graph, due to some triangular graph that may fulfil this criterion, it remains strongly indicative of the pole character of the process under consideration.

The Treiman-Yang (TY) criterion establishes that the amplitude of the 


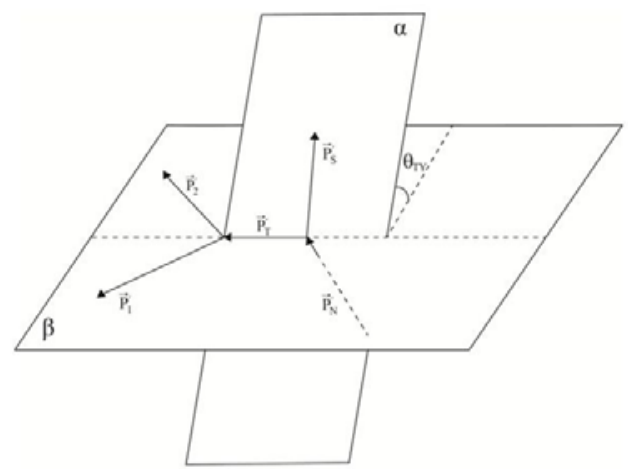

Figure 2: TY criterion as the rotation of plane $\alpha$ with respect to plane $\beta$.

three-body reaction should be invariant under rotation of the $\left(\overrightarrow{p_{1}}, \overrightarrow{p_{2}}\right)$ plane around the sum of these momenta, in a reference frame in which the projectile is at rest [2]. The quantities of three Galilean invariants do not change if in the anti-laboratory system (that is the system for which $\overrightarrow{p_{0}}=0$ ) the momenta of the particles 1 and 2 are rotated with respect to their sum. In these condition the differential cross section must remain constant under this rotation.

The application of the Treiman-Yang criterion is related to the comparison of the differential cross-sections measured at different detection conditions corresponding to the same value of the Galilean invariants but with differents angles $\Theta_{T Y}$. In particular a Treiman-Yang distribution can be visualized as the rotation of the plane $\alpha$ with respect to plane $\beta$ as shown in fig.2.

The plane $\alpha$ is defined by $\overrightarrow{p_{T}^{\prime}}$ and $\overrightarrow{p_{N}^{\prime}}$, the plane $\beta$ by $\overrightarrow{p_{T}^{\prime}}$ and $\overrightarrow{p_{1}^{\prime}}$ :

$\cos \Theta_{T Y}=\frac{\left.\left.\overrightarrow{\left(p_{T}^{\prime}\right.} \times \overrightarrow{p_{N}^{\prime}}\right) \bullet \overrightarrow{p_{T}^{\prime}} \times \overrightarrow{p_{1}^{\prime}}\right)}{\left|\overrightarrow{p_{T}^{\prime}} \times \overrightarrow{p_{N}^{\prime}}\right| \bullet|\cdot| \overrightarrow{p_{T}^{\prime}} \times \overrightarrow{p_{1}^{\prime}} \mid}$

And by taking into account the identity:

$(\vec{A} \times \vec{B}) \bullet(\vec{C} \times \vec{D})=(\vec{A} \bullet \vec{C}) \bullet(\vec{B} \bullet \vec{D})-(\vec{A} \bullet \vec{D}) \bullet(\vec{B} \bullet \vec{C})$

It can be shown that

$\cos \Theta_{T Y}=\frac{p_{T}^{\prime} p_{1 z}^{\prime}+p_{T z}^{\prime}\left(p_{T x}^{\prime} p_{1 x}^{\prime}+p_{T y}^{\prime} p_{1 y}^{\prime}+p_{T z}^{\prime} p_{1 z}^{\prime}\right)}{\sqrt{{p_{T y}^{\prime}}^{2}+p_{T x}^{\prime}} \cdot \sqrt{\left(p_{T y}^{\prime} p_{1 z}^{\prime}-p_{T z}^{\prime} p_{1 y}^{\prime}\right)^{2}+\left(p_{T z}^{\prime} p_{1 x}^{\prime}-p_{T x}^{\prime} p_{1 z}^{\prime}\right)^{2}+\left(p_{T x}^{\prime} p_{1 y}^{\prime}-p_{T y}^{\prime} p_{1 x}^{\prime}\right)^{2}}}$

Where all the momenta are in the antilaboratory system. The transformation from laboratory to the antilaboratory system is:

$\overrightarrow{p_{k}^{\prime}}=\overrightarrow{p_{k}}-\frac{m_{k}}{m_{0}} \overrightarrow{p_{0}}$

In the case of the present work, we want to extend and experimentally proof the pole approximation validity by means of the TY criterion applied 


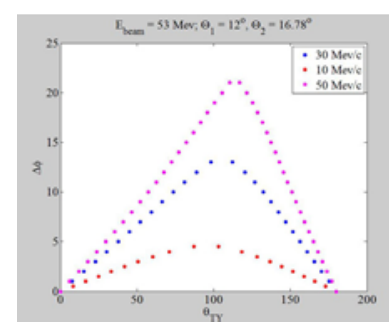

a)

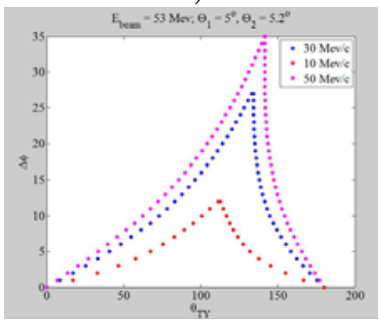

b)

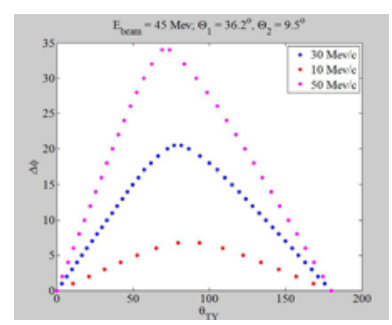

c)

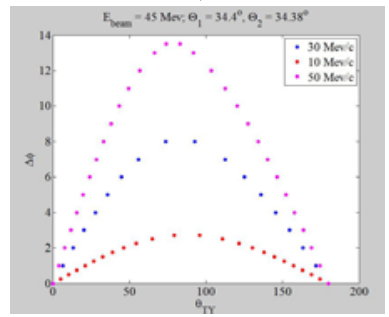

d)

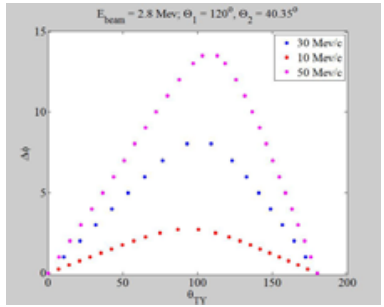

e)

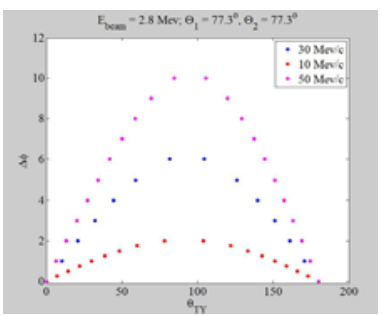

f)

Figure 3: Correlation between $\phi_{2}$ and $\Theta_{T Y}$ angles for various fixed value of the spectator momentum modulus

to three cases:

- $H^{2}\left(B^{10}, \alpha B e^{7}\right) n_{s}$ reaction induced at a boron beam energy of $53 \mathrm{MeV}$ in the laboratory(fig.3.a, fig.3.b). The plan is to use the $p+n_{s}$ cluster configuration of the target $H^{2}$ nucleus and detect the emitted $B e^{7}$ and $\alpha$ particles in the binary virtual reaction $H^{1}\left(B^{10}, B e^{7}\right) \alpha$.

- $H^{1}\left(B^{11}, \alpha_{1} \alpha_{2}\right) \alpha_{s}$ reaction induced at a boron beam energy of $45 \mathrm{MeV}$ in the laboratory(fig.3.c, fig 3.d). The plan is to use the $\alpha_{s}+L i^{7}$ cluster configuration of the projectile $B^{11}$ nucleus and detect the emitted $\alpha_{1}$ and $\alpha_{2}$ particles in the binary virtual reaction $H^{1}\left(L i^{7}, \alpha_{1}\right) \alpha_{2}$.

- $H e^{3}\left(B e^{9}, \alpha_{1} \alpha_{2}\right) \alpha_{s}$ reaction induced at a helium beam energy of 2.8 $\mathrm{MeV}$ in the laboratory(fig.3.e, fig.3.f). The plan is to use the $H e^{5}+\alpha_{s}$ cluster configuration of the target $B e^{9}$ nucleus and detect the emitted $\alpha_{1}$ and $\alpha_{2}$ particles in the binary virtual reaction $H e^{3}\left(H e^{5}, \alpha_{1}\right) \alpha_{2}$.

\section{References}

[1] R.Tribble, C. Bertulani, M. La Cognata, A. Mukhamedzhanov, C. Spitaleri, Indirect techniques in nuclear astrophysic, Rep. Prog. Phys. 77 (2014) 106901 (49pp).

[2] S. B. Treiman and C. N. Yang, Phys. Rev. Lett. 8 (1962) 140. 